\title{
Passive smoke exposure and type-2 diabetes: is it time for action?
}

\author{
Fabrizio Faggiano • Elias Allara
}

Received: 17 June 2014/ Accepted: 11 September 2014/Published online: 20 September 2014

(C) Springer Science+Business Media New York 2014

In a recent meta-analysis [1] of six cohort studies, Sun et al. found evidence of a $21 \%$ increased risk of type-2 diabetes or glucose intolerance among subjects exposed to passive smoking (95\% CI 7-38\%) compared to those who were not exposed. The authors conclude that such association may have a 'far-reaching significance' for public health. At a first glance, such conclusion might be justified. The metaanalysis appears to have good methodological quality, including an adequately powered pooled sample size $(n=154,406)$ and using a random effects model to address between study heterogeneity.

Association, however, does not necessarily imply causation. Findings might be due to alternative explanations, although the large pooled sample size minimises the risk for random error. First, since the studies included in the meta-analysis are observational, passive smoking exposure could mask the effect of other causal factors of diabetes such as being overweight or obese, diet habits, and family history-and adjustments performed in individual studies may have still left considerable residual confounding. Second, assessment of the exposure could be biased and overestimated among those with diabetes due to lack of blinding of the assessors.

\footnotetext{
F. Faggiano - E. Allara

Department of Translational Medicine, UPO - Università del

Piemonte Orientale, Novara, Italy

F. Faggiano $(\bowtie)$

Department of Translational Medicine, Avogadro University, Via Solaroli 17, 28100 Novara, Italy

e-mail: fabrizio.faggiano@med.unipmn.it

E. Allara

School of Public Health, University of Torino, Turin, Italy
}

Ascertaining whether or not an observed association estimate accurately represents the underlying population parameter is probably one of the most challenging tasks for epidemiologists, especially when the association is relatively weak such in Sun et al.'s meta-analysis. A thorough application of the process of reasoning elucidated by Sir Austin Bradford-Hill (1897-1991), and already cited by the authors, may be helpful [2]. Despite being first presented nearly 50 years ago, Hill's nine criteria of causation are still widely accepted. Such multifaceted approach might be helpful in summarizing the intrinsic and contextual factors involved in the link between an exposure and a disease, especially with a relatively weak association.

For the association between passive smoking and diabetes, Hill's criteria may be applied as follows:

1. Strength of the association. Some causal factors are strongly associated with some diseases, e.g., tobacco smokers have about ten times greater risks of lung cancer compared to non-smokers, and asbestos workers have several dozen times greater risk of developing malignant mesothelioma $[3,4]$. Sun et al. found a 1.21 greater risk of diabetes among those exposed to passive smoke, which is relatively small-albeit non-negligible-strength of association.

2. Consistency, i.e., the coherence among repeated observations. Point estimates of all of the six included studies go in the same direction, and there is an overlap of 5 out of 6 confidence intervals. Additionally, the findings of the meta-analysis are consistent with those of two other systematic reviews. Both Wang et al. [5], which pooled the findings of four cohort studies with an overall sample of 112,351 subjects, and Zhu et al. [6], which included 14 studies, came up to similar conclusions. 
3. Specificity. According to Bradford-Hill, the exposure under consideration should be the unique (rare in medicine) or predominant cause of the disease of interest. This criterion, close to the paradigm of infectious diseases, is hardly applicable to diabetes, which is a multifactorial disease.

4. Temporality. The confidence that exposure precedes disease is particularly important when studying diseases with slow development such as cancer and diabetes. However, temporality does not seem of particular concern in this case. Sun et al. included only prospective cohort studies, which allow distinguishing between time of exposure to passive smoking and time of diabetes onset.

5. Biological gradient, i.e., the presence of a relationship between amount of exposure and risk of disease, is a strong element to infer causation. This criterion seems to be met in the work by Sun et al. The authors observed a dose-response effect ranging from a risk ratio of 1.19 (95\% CI $0.96-1.47$ ) of developing diabetes for subjects exposed to less than $1 \mathrm{~h}$ per day of passive smoke, to a risk ratio of 1.48 (95\% CI 1.17-1.86) for subjects exposed to four or more hours per day of passive smoke.

6. Biological plausibility is not essential according to Bradford-Hill, as epidemiology has sometimes anticipated the understanding of biological mechanisms. In 1854, when John Snow noted a cluster of cholera cases around a water pump located in Soho, London, the etiological agent of cholera was still unknown. In the case of passive smoke and diabetes, we are not aware of direct biological evidence. However, is seems reasonable that directly inhaled smoke and secondhand smoke may act through similar biological mechanisms. A recent report of the U.S. Surgeon General [3] states that there is sufficient evidence to infer a causal relationship between active smoking and diabetes. Such statement is supported by a considerable body of both epidemiological and biological evidence: (i) smoking is independently associated to central obesity, which is a risk factor for insulin resistance and diabetes, via a number of biological pathways (higher concentrations of fasting plasma cortisol; greater stimulation of the sympathetic nervous system; and lower testosterone levels in men); (ii) smoking increases inflammatory markers and oxidative stress, and impairs endothelial function, which are all implicated in the development of insulin resistance and impairment of glucose function; (iii) smoking can reduce insulin release through neuronal nicotinic acetylcholine receptors on pancreatic islet cells (in both human and animal models) and by causing dysfunction and apoptosis of beta cells mediated via the mitochondrial and/or death receptor pathway (in animal models). According to the same report, active smoking is held responsible for $37 \%$ greater risk of diabetes [3], which is only marginally greater than the $21 \%$ greater risk of diabetes found by Sun et al. for passive smoke. Such findings can hardly be considered proportional to exposure. For example, in the association between tobacco smoking and lung cancer the estimated risk ratio for active smoking is approximately 11.7 [7], whereas the risk ratio for passive smoking ranges from 1.15 to 1.60 [8].

7. Coherence. The interpretation of the association between passive smoking and diabetes does not appear to conflict with the generally known facts of the natural history and biology of diabetes, as described in the previous paragraph.

8. Experiment. According to Bradford-Hill, the strongest support for causation may derive from experimental studies, which for obvious ethical reasons cannot be carried out in humans to assess the effects of exposure to risk factors. Besides considering studies involving animal models exposed to tobacco smoke, which are in favor of a causal relationship with diabetes, we can appraise the methodological robustness of the observational studies included in Sun et al.'s meta-analysis to imply how close they may be to an ideal experimental study. For example, adjustment for confounding is a key element of study quality. Sun et al. noted that only one study adjusted for ethnicity [9], and one study [10] failed to adjust for any confounder. However, most of the studies controlled for the most common confounders. Four out of six studies adjusted for an indicator of socio-economic status [9, 11-13]; four out of six studies adjusted for family history of diabetes [9, 11, 12, 14]. Sun et al. also used a random effects model to address study heterogeneity, thus accounting for differences in the cultural contexts in which the studies were carried out. In general, we feel that the studies included by Sun et al. and the methods used to pool their estimates are adequate. However, it is worth noting that the cohort study design of the included studies, despite being probably the most indicated for studying the association between passive smoking and diabetes, does not allow controlling for unknown confounders which therefore cannot be excluded a priori.

9. Analogy. Other air pollutants are known to be associated with type- 2 diabetes mellitus. There is epidemiological evidence that exposure to ambient particulate matter (PM) may increase markers of insulin sensitivity and diabetes prevalence. Experimental studies found that PM exposure (i) alters endothelial function in both animals and humans, and that such alterations 
may result in reduced glucose uptake; (ii) is associated with increased systemic pro-inflammatory markers, an increase in adipose tissue macrophages, and oxidant stress; (iii) is implicated in developing defective insulin signaling in the liver, which is fundamental to the pathogenesis of insulin resistance and diabetes mellitus. However, the comparison with the effects of active and second-hand tobacco smoking on lung cancer may suggest some caution. As mentioned above, the ratio between the risk ratio for active smoking and the risk ratio for passive smoking is 7-10, whereas in the case of diabetes it is 1.13 . While we could assume that the differential effect of passive and active smoking on lung cancer is mainly a matter of dose, is it credible for diabetes?

In conclusion, several elements suggest causal relationship between passive smoking and diabetes, in particular the fulfillment of the temporality criterion, the presence of an apparent dose-response effect, and the indirect plausibility of the biological effect, as well as the analogy with PM exposure. However, the relative weakness of the association and the small difference between the risk ratios for active and passive smoking may suggest some caution.

Large epidemiological studies with sufficient power and long follow-up periods are needed, as well as studies specifically exploring the effects of second-hand smoking on the functioning of biological pathways.

Nonetheless, the study by Sun et al. can already has a prominent role for public health. On a global scale, 350 million people are affected by diabetes [15], causing the loss of 46.9 million disability-adjusted life years (DALYs)-more than the loss of 32.4 million DALYs due to lung cancer [16]. The suspect of a causal relationship between passive smoking and diabetes can further encourage the global efforts toward tobacco-free public places by advocating more policy actions to protect the population from the hazard of second-hand smoke and to promote $100 \%$ indoor smoke-free environments [17].

\section{References}

1. K. Sun, D. Liu, C. Wang, M. Ren, C. Yang, L. Yan, Passive smoke exposure and risk of diabetes: a meta-analysis of prospective studies. Endocrine. (2014). doi:10.1007/s12020-0140194-1

2. A. Bradford-Hill, The environment and disease: association or causation? Proc. R. Soc. Med. 58, 295-300 (1965)
3. U.S. Department of Health and Human Services, The Health Consequences of Smoking-50 Years of Progress. A Report of the Surgeon General (U.S. Department of Health and Human Services, Atlanta, 2014)

4. B. Charbotel, B. Fervers, J.P. Droz, Occupational exposures in rare cancers: a critical review of the literature. Crit. Rev. Oncol. Hematol. 90, 99-134 (2014)

5. Y. Wang, J. Ji, Y. Liu, X. Deng, Q. He, Passive smoking and risk of type 2 diabetes: a meta-analysis of prospective cohort studies. PLoS ONE 8, e69915 (2013)

6. B. Zhu, X. Wu, X. Wang, Q. Zheng, G. Sun, The Association between passive smoking and type 2 diabetes: a meta-analysis. Asia Pac. J. Public Health 26, 226-237 (2014)

7. International Agency for Research on Cancer (IARC), Tobacco smoking, in IARC. A Review of Human Carcinogens. Part E: Personal Habits and Indoor Combustions IARC Monographs, vol 100E (Lyon, France, 2012)

8. International Agency for Research on Cancer (IARC), Secondhand tobacco smoke, in IARC. A Review of Human Carcinogens. Part E: Personal Habits and Indoor Combustions IARC Monographs, vol 100E (Lyon, France, 2012)

9. L. Zhang, G.C. Curhan, F.B. Hu, E.B. Rimm, J.P. Forman, Association between passive and active smoking and incident type 2 diabetes in women. Diabetes Care 34, 892-897 (2011)

10. T.K. Houston, S.D. Person, M.J. Pletcher, K. Liu, C. Iribarren, C.I. Kiefe, Active and passive smoking and development of glucose intolerance among young adults in a prospective cohort: CARDIA study. BMJ 332, 1064-1069 (2006)

11. B. Kowall, W. Rathmann, K. Strassburger, M. Heier, R. Holle, B. Thorand, G. Giani, A. Peters, C. Meisinger, Association of passive and active smoking with incident type 2 diabetes mellitus in the elderly population: the KORA S4/F4 cohort study. Eur. J. Epidemiol. 25, 393-402 (2010)

12. M. Lajous, L. Tondeur, G. Fagherazzi, B. de Lauzon-Guillain, M.-C. Boutron-Ruaualt, F. Clavel-Chapelon, Childhood and adult secondhand smoke and type 2 diabetes in women. Diabetes Care 36, 2720-2725 (2013)

13. K.-P. Ko, H. Min, Y. Ahn, S.-J. Park, C.-S. Kim, J.K. Park, S.S. Kim, A prospective study investigating the association between environmental tobacco smoke exposure and the incidence of type 2 diabetes in never smokers. Ann. Epidemiol. 21, 42-47 (2011)

14. Y. Hayashino, S. Fukuhara, T. Okamura, H. Yamato, H. Tanaka, T. Tanaka, T. Kadowaki, H. Ueshima, A prospective study of passive smoking and risk of diabetes in a cohort of workers: the High-Risk and Population Strategy for Occupational Health Promotion (HIPOP-OHP) study. Diabetes Care 31, 732-734 (2008)

15. G. Danaei, M.M. Finucane, Y. Lu, G.M. Singh, M.J. Cowan, C.J. Paciorek, J.K. Lin, F. Farzadfar, Y.-H. Khang, G.A. Stevens, M. Rao, M.K. Ali, L.M. Riley, C.A. Robinson, M. Ezzati, National, regional, and global trends in fasting plasma glucose and diabetes prevalence since 1980: systematic analysis of health examination surveys and epidemiological studies with 370 country-years and 2.7 million participants. Lancet 378, 31-40 (2011)

16. Institute for Health Metrics and Evaluation, GBD Compare, http://viz.healthmetricsandevaluation.org/gbd-compare/

17. World Health Organization (WHO), Protection from exposure to second-hand tobacco smoke. Policy recommendations (World Health Organization (WHO), Geneva, 2007) 